\title{
Exosomes derived from SIRT6 transfected urine-derived stem cells improves renal fibrosis
}

Rui Wang ${ }^{1 *}$, Weijie Xie ${ }^{1}$, Jiawei Zhang ${ }^{1}$, Huan Guo ${ }^{1}$

1Dept. of Urology, Shenzhen University General Hospital, Shenzhen University, Shenzhen, 518000, PR China

${ }^{*}$ Correspondence: Rui Wang

NO.1098, Xueyuan Road, Shenzhen University City, Nanshan District, Shenzhen, 518000, PR China

Email: 18664563718@163.com

\section{Abstract:}

Purpose: The treatment of renal fibrosis caused by long-term obstructive nephropathy is limited. The purpose of this study was to establish a mouse model of renal fibrosis with unilateral ureteral obstruction (UUO) and to treat it with exosomes derived from SIRT6 transfected urine-derived stem cells (USCs), which will determine whether exosomes have the effect of anti-fibrosis Methods: The renal fibrosis model of UUO mice was established by ligating unilateral ureter. USCs were extracted from human urine and transfected with SIRT6 lentivirus plasmid. The SIRT6-USCs-exosomes(SIRT6-USCs-exos) were collected and identified by transmission electron microscope, nanoparticle tracking analysis and western blot. SIRT6-USCs-exos were injected into the tail vein of mice and the renal tissue of mice was stained with HE. The relative gene expressions of $\alpha$-SMA, E-cadherin and TGF- $\beta 1$ were analyzed by RT-qPCR.

Results: In this study, we successfully constructed the renal fibrosis model of UUO mice and hUSCs with overexpression of SIRT6. The SIRT6-USCs-exos significantly improved the renal fibrosis in UUO mice. The relative mRNA 
expressions of fibrosis-related genes $\alpha-S M A$, E-cadherin and TGF- $\beta 1$ in renal tissue were significantly down-regulated after SIRT6-USCs-exos treatment. Conclusion: Our results indicate that SIRT6-USCs-exos can effectively alleviate renal fibrosis caused by long-term obstructive nephropathy. The findings may be promising for dealing with renal fibrosis.

Keywords: renal fibrosis, USCs, exosome, SIRT6

\section{Introduction}

Obstructive nephropathy refers to the obstruction of renal pelvis, ureter, bladder and urethra caused by a variety of reasons, which is common in urinary tract obstruction caused by urinary calculi, benign prostatic hyperplasia, neurogenic bladder, abnormal urinary tract anatomy in children and so on. In recent years, the diseases of urinary calculi and benign prostatic hyperplasia show a rising and complicated trend in clinic[1,2]. Long-term urinary tract obstruction caused by these diseases reduces the blood supply of renal tissue, resulting in renal tubular cell atrophy and interstitial fibrosis. Chronic progressive renal fibrosis can lead to irreversible renal failure, gradually decline the quality of life of patients, and cause a huge medical burden to the society. At present, for the renal fibrosis caused by long-term obstructive nephropathy, surgery can relieve the obstruction, but can not further improve the renal fibrosis. Therefore, to find out an effective treatment of renal fibrosis is an urgent clinical problem to be solved.

Stem cells can provide tissue regeneration and promote recovery in most injuries because of their unique ability to replicate and differentiate into special organ cells. They can be divided into embryonic stem cells and somatic stem cells according to the stage of development. At present, mesenchymal stem cells (MSCs) are the most widely studied in experimental biology and medicine, which belong to body stem cells and exist in human mesoderm tissue, including amniotic fluid, umbilical cord tissue, bone marrow, adipose tissue, 
testis and so on. Urine-derived stem cells (USCs) extracted from human urine can express a variety of renal cortical markers and the same cellular markers as MSCs, so they are considered to be a kind of mesenchymal stem cells derived from renal[3]. Compared with MSCs that need to be extracted from bone marrow or fat, the sample collection process and cell separation methods of USCs are simple, non-invasive, and have few ethical issues, making the clinical application prospects of USCs more advantageous[4].

Stem cells interact between cells by secreting exosomes with a molecular diameter of $30-100 \mathrm{~nm}$. Exosomes contain a variety of biologically active molecules, such as cytokines, signal peptides, microRNAs, lipids and DNA, which are regarded as an important bridge for the communication between cells. More and more studies have shown that USCs can promote the repair of damaged renals $[5,6]$, delay diabetic nephropathy and improve diabetic erectile dysfunction[7] through a variety of molecules in exosomes. Recent studies have shown that USCs can inhibit the fibrosis of cardiomyocytes, glomerular cells and bladder detrusor in a rat model of type 2 diabetes[8]. However, the therapeutic effect of USCs exosomes (USCs-exos) alone is limited. How can the therapeutic effect of USCs be improved?

With the continuous development of genetic engineering, research on genetic modification of stem cells has gradually increased. The stem cells can be transfected with therapeutic genes or therapeutic factors, and then target to the lesion site through stem cell exosomes, so as to achieve better treatment effects. A large number of studies have shown that gene-transfected stem cell exosomes play an important role in improving the fibrosis of the liver[16], tendons[9], and myocardium[10]. This technology not only enriches the treatment methods of stem cells, but also expands the scope of application of stem cell therapy. 
Sirtuins are an evolutionarily conserved family of deacetylases that depend on nicotinamide adenine dinucleotide (NAD+), which are associated with proliferation, DNA repair, mitochondrial energy homeostasis, and antioxidant activity. Studies have shown that SIRT6 attenuates or inhibits the fibrosis process of heart[11,12], lung[13,14], liver[15,16] and renal $[17,18]$ by affecting cell differentiation, TGF- $\beta 1 /$ Smads signal pathway and NF-KB signal pathway. Therefore, as an excellent anti-fibrosis factor, we chose SIRT6 to transfect USCs and obtain SIRT6-USCs-exos.

In this study, we found exosomes derived from SIRT6 transfected urine-derived stem cells (SIRT6-USCs-exos) inhibited renal fibrosis of UUO mice. RT-qPCR showed the relative mRNA expressions of fibrosis-related genes $\alpha-S M A, E-c a d h e r i n$ and TGF- $\beta 1$ in renal tissue were significantly down-regulated after SIRT6-USCs-exos treatment.

\section{Methods}

\section{Animal model}

One week after adaptation, the mice were randomly divided into four groups: sham operation group, unilateral ureteral obstruction (UUO) group and GFP-USCs-exos group and SIRT6-USCs-exos group. The right ureter was ligated and cut through the right back incision in mice, but only free ureter was not ligated in Sham group. The experimental animals were given drugs three days before the establishment of the model. 10ugl SIRT6-USCs-exos was injected into the tail vein of rats in the SIRT6-USCs-exos group for one week. 10ugl GFP-USCs-exos was injected into the tail vein of rats in the GFP-USCs-exos group for one week. The mice were killed on the 7 th day after the establishment of the model, and the serum and renal tissue samples were taken.

\section{Cell procedure}


Urine samples of healthy adult volunteers (22-35 years old) were collected, and all volunteers signed informed consent forms. Under aseptic condition, $200 \mathrm{~mL}$ fresh midstream urine of healthy adult volunteers was collected in a disposable aseptic container, divided into 4 tubes with a $50 \mathrm{~mL}$ centrifuge tube and centrifuged for 10 minutes. Then the supernatant was absorbed and discarded. PBS containing $1 \%$ penicillin streptomycin was added to the supernatant and washed evenly. After repeated centrifugation, the supernatant was discarded. Finally, the remaining sediment was re-suspended with a special culture medium for $5 \mathrm{~mL}$ urine-derived stem cells and inoculated on a 24-well plate. After 3 days of static culture in a $5 \% \mathrm{CO}_{2}$ incubator at $37^{\circ} \mathrm{C}$, the cell growth was observed, the medium was changed, and the non-adherent cells were discarded, and then the liquid was changed every 3 days. When the primary cultured urine-derived stem cells reached $80 \%$ of $90 \%$ fusion, they were digested with $0.25 \%$ trypsin and inoculated at $1: 2$ ratio. The morphological changes of the cells were observed under inverted microscope.

\section{Isolation and identification of exosomes}

At $80-90 \%$ confluence, USCs were cultured in vesicle-depleted medium for 48-72 hours. The medium was gathered and subjected to gradient centrifugation. Briefly, the medium was first centrifuged at $1000 \mathrm{~g}$ for $10 \mathrm{~min}$, at $3000 \mathrm{~g}$ for $30 \mathrm{~min}$, at $10,000 \mathrm{~g}$ for $60 \mathrm{~min}$, at $100,000 \mathrm{~g}$ for 4 hours. All centrifugal steps were performed at $4{ }^{\circ} \mathrm{C}$. Exosomes were stored at $-80^{\circ} \mathrm{C}$ or used for downstream experiments. Transmission electron microscopy (TEM), nanoparticle tracking analysis (NTA) and western blotting were used to identify the collected exosomes.

\section{USCs transfection, selection, and SIRT6 expression}

A green fluorescent protein (GFP) label for a lentiviral vector plasmid system carrying the SIRT6 gene was constructed by Shanghai Jikai Gene Technology Co., Ltd. USCs were transfected with lentiviral vectors at an appropriate 
multiplicity of infection $(\mathrm{MOI}=20)$ according to the manufacturers' instructions. GFP expression was observed via fluorescence microscopy at 1,3 , and 5 days after lentiviral vector transfection. The protein expression of SIRT6 in USCs after SIRT6 transfer was verified by western blot. The protein expression of SIRT6 in both SIRT6-USCs-exos and GFP-USCs-exos was measured by western blot.

\section{Western blot}

Cells were lysed in RIPA buffer ( $1 \%$ NP- $40,0.5 \%$ sodium deoxycholate, $0.1 \%$ SDS in PBS) containing a Complete Protease Inhibitor Cocktail. Protein concentration was determined by Bio-Rad DC protein assay. Total protein (30 ug) from the cell lysate was separated by SDS-PAGE and transferred to a nitrocellulose membrane. The membrane was blocked in $5 \%$ non-fat milk in PBS overnight and then incubated with primary antibody at $37^{\circ} \mathrm{C}$ for $2 \mathrm{~h}$. After washing for $30 \mathrm{~min}$, the membrane was incubated with 1:3000 secondary goat anti-mouse IgG in TBS-Tween for $1 \mathrm{~h}$ at $37{ }^{\circ} \mathrm{C}$. After further washes, the proteins of interest were detected using a Chemiluminescent HRP Antibody Detection Kit and the signals were captured using an electro chemiluminescent system. The anti-CD63, anti-TSG1001, anti-ALIX and anti-SIRT6 antibody was used at a 1:1000 dilution, the anti-GAPDH was used at a 1:1500 dilution.

\section{RT-qPCR}

Total RNA was extracted from renal tissues and cells using TRIzol reagent according to the manufacturer's instructions (Invitrogen, USA), and one microgram of RNA was reverse transcribed to first-strand cDNA using the GoScript reverse transcription system (Promega, USA). Quantitative PCR was conducted using SYBR master mix (Invitrogen, USA) on a Roche LightCycler 480II. Relative mRNA expression levels were calculated using the $2^{-\Delta \Delta C t}$ method and were normalized to the corresponding expression levels of 
GAPDH. The primer sequences used to amplify the mouse RNA are shown as follow:

a-SMA: Forward primer: 5'-GCCTTGGTGTGTGACAATGG-3'; Reverse primer: 5'-AAAACAGCCCTGGGAGCAT -3'

E-cadherin: Forward primer: 5'-TGCCCAGAAAATGAAAAAGG-3'; Reverse primer: 5'-GTGTATGTGGCAATGCGTTC -3'

TGF-ß1: Forward primer: 5'-GTAGCTCTGATGAGTGCAATGAC-3'; Reverse primer: 5'-TATTGCCAGTCACGCATGCTTAGC-3'

GAPDH: Forward primer: 5'-AGGTCGGTGTGAACGGATTTG-3'; Reverse primer: 5'-GGGGTCGTTGATGGCAACA-3'

\section{Results}

\section{Characterization of exosomes}

We successfully constructed USCs that stably overexpressed SIRT6 and collected exosomes derived from USCs. The exosomes purified from USCs medium were identified by transmission electron microscope (TEM). The diameter of exosomes was about 100nm (Fig. 1A). Then the expression of exosome markers CD63, TSG101 and ALIX was confirmed by Western blot (Fig. 1B). Next, the concentration and size distribution of exocrine bodies were analyzed by NanoSightLM10 Nanoparticle Tracking Analysis (NTA). In USCs medium, the concentration of exosome in control group was $8.42 \times 10^{8} / \mathrm{ml}$, and that in overexpression SIRT6 group was $4.39 \times 10^{8} / \mathrm{mL}$. The number of exocrine bodies in USCs medium overexpressing SIRT6 was lower than that in the control group, and NTA estimated that the size of vesicles was between 60 and 140nm (Fig. 1C). Finally, the expression of SIRT6 in the exosome secreted by USCs was identified by Western blot. The results showed that compared with the exosome of the control USCs, the USCs overexpressing SIRT6 and the SIRT6 contained in the exosome were significantly increased (Fig. 1D). 
A

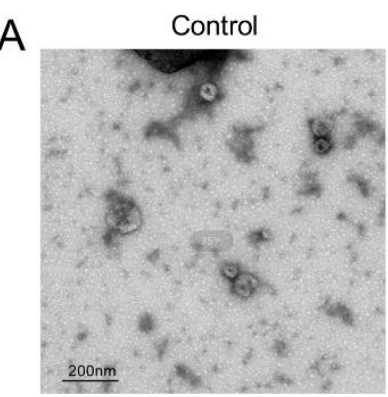

C

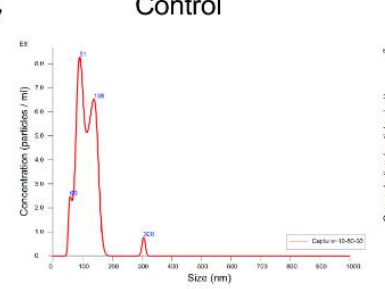

over-Sirt6

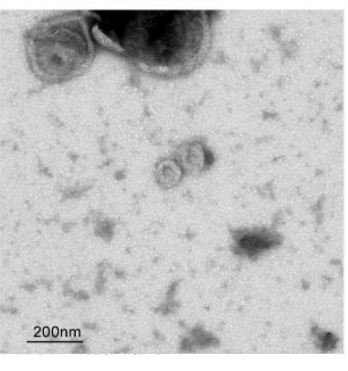

over-Sirt6
B

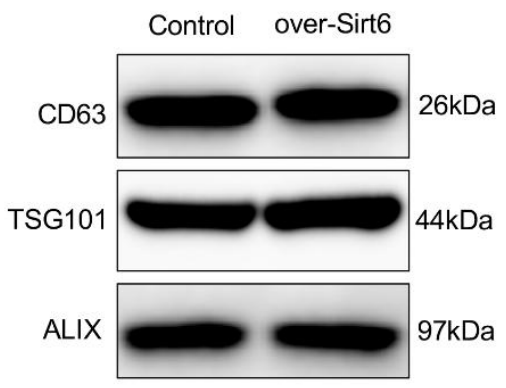

$\mathrm{D}$

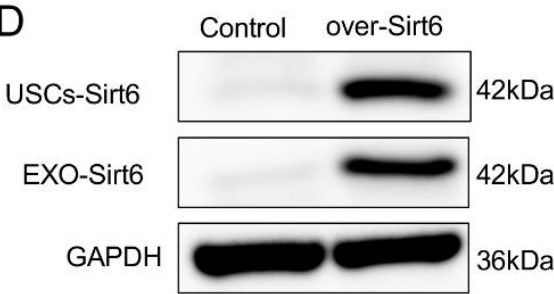

Figure.1. USCs and SIRT6-USCs-exos characterization. A: exosome morphology under transmission electron microscope. B: Western blot showed that CD63,TSG101 and ALIX were expressed in both USCs-exos and SIRT6-USCs-exos. C: NTA results showed the number of exocrine bodies in USCs and SIRT6-USCs culture medium, respectively. D: Western blot showed that the expression level of SIRT6 in SIRT6-USCs and SIRT6-USCs-exos was significantly higher than that in USCs and USCs-exos.

\section{SIRT6-USCs-exos effectively alleviated renal fibrosis in UUO mouse model}

HE staining results showed that SIRT6-USCs-exos treatment significantly alleviated renal fibrosis compared with the other three groups. Although the GFP-USCs-exos also reduced the collagen deposition in the renal tissue, the improvement effect was not as good as that of the SIRT6-USCs-exos group (Fig. 2A). Further RT-qPCR analysis demonstrated that the gene expressions of $\alpha$-SMA, E-cadherin and TGF- $\beta 1$ were significantly upregulated in the UUO group compared with the sham group. Similarly, the gene expressions of $\alpha-S M A, E-c a d h e r i n$ and TGF- $\beta 1$ were significantly downregulated in both GFP-USCs-exos and SIRT6-USCs-exos groups. Moreover, the anti-fibrosis effect of the SIRT6-USCs-exos treatment was more significant. The above results showed that the USCs-exos could improve renal fibrosis by 
downregulating the expression of fibrosis-related genes, but the therapeutic effect of the SIRT6-USCs-exos was better.

A

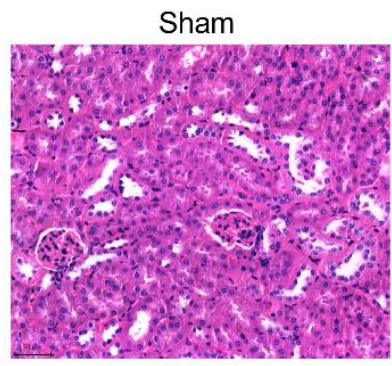

GFP-USCs-exos

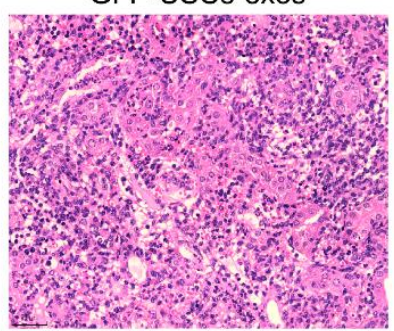

B

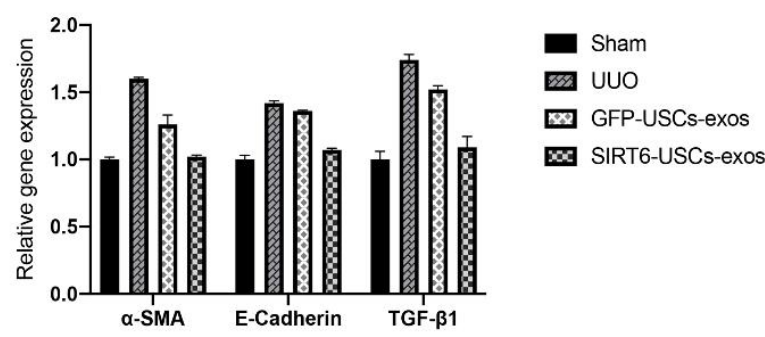

Figure.2 HE staining and RT-qPCR analyses of renal tissue in mice model. A. HE staining of mice renal tissue in different groups showed different degrees of fibrosis. B. The RT-qPCR analyses showed the relative gene expression of $\alpha$-SMA, E-cadherin and TGF- $\beta 1$ of mice renal tissue in different groups.

\section{Discussion}

Cell-based regenerative therapy has been widely used in patients who have no other treatment options as an alternative treatment. More and more evidences show that stem cells have a better effect on improving damage and fibrosis. In this study, we demonstrated that administration of USCs-exos on UUO model ameliorated tubular interstitial fibrosis. We further used SIRT6-USCs-exos to show better repair ability of renal fibrosis in UUO mice than GFP-USCs-exos. 
Although we have observed in this experiment that both urine-derived stem cells and modified urine-derived stem cells can improve renal fibrosis, the specific mechanism is still unclear. Previous studies have shown that USCs can promote renal damage and repair through a variety of molecules in exosomes, for example, microRNA[5,6]. Recent studies have shown that USCs can inhibit the fibrosis of cardiomyocytes, glomerular cells and bladder detrusor in a rat model of type 2 diabetes[8].

The TGF- $\beta$ signaling pathway is a key mediator involved in the process of organ fibrosis, and TGF- $\beta 1$ is considered to be an important cytokine for the transition from inflammatory diseases to fibrosis[19,20]. Studies have found that bone marrow-derived exosomes can regulate fibrosis repair of damaged endometrium by down-regulating TGF- $\beta 1[21]$. In this study, mouse ureteral obstruction caused high expression of TGF- $\beta 1$ and $\alpha-S M A$ in renal tissue. After treatment with SIRT6-USCs-exo, the expression of fibrosis-related proteins TGF- $\beta 1$ and $\alpha-S M A$ could be down-regulated.

In summary, this study shows that SIRT6-USCs-exos can effectively alleviate renal fibrosis induced by UUO in mouse, but whether it can inhibit human renal fibrosis remains to be confirmed. In addition, this experiment is aimed at the interventional treatment in the early stage of renal fibrosis, and whether there is a relieving effect on the renals in the middle and late stages of failure requires further experiments.

\section{Conflicts of interest}

All authors declare that there is no conflict of interest associated with this manuscript.

\section{Reference}

1. Rui W, Hongbo S, Ji Z, Jun C, Xiaoli L, Chen D: Clinical effect of 
single-tract percutaneous nephrolithotomy and flexible ureterorenoscopy in the second stage treatment of complicated renal calculi. Journal of Minimally Invasive Urology 2018, 7(02):101-104.

2. Rui W, Chen D, Xiaoli L, Bo X, Ji Z, Xiaosong S, Hongbo S: Clinical effect of transurethral plasmakinetic resection of prostate for benign prostatic hyperplasia with acontractile detrusor. Journal of Minimally Invasive Urology 2016, 5(06):364-366.

3. Bento G, Shafigullina AK, Rizvanov AA, Sardão VA, Macedo MP, Oliveira PJ: Urine-Derived Stem Cells: Applications in Regenerative and Predictive Medicine. Cells 2020, 9(3):573.

4. Pavathuparambil Abdul Manaph N, Al-Hawwas M, Bobrovskaya L, Coates PT, Zhou X-F: Urine-derived cells for human cell therapy. Stem Cell Res Ther 2018, 9(1):189.

5. Zhang Y, Wang J, Yang B, Qiao R, Li A, Guo H, Ding J, Li H, Ye H, Wu D et al: Transfer of MicroRNA-216a-5p From Exosomes Secreted by Human Urine-Derived Stem Cells Reduces Renal Ischemia/Reperfusion Injury. Front Cell Dev Biol 2020, 8:610587-610587.

6. Li X, Liao J, Su X, Li W, Bi Z, Wang J, Su Q, Huang H, Wei Y, Gao Y et al: Human urine-derived stem cells protect against renal ischemia/reperfusion injury in a rat model via exosomal miR-146a-5p which targets IRAK1. Theranostics 2020, 10(21):9561-9578.

7. Yang Q, Chen W, Han D, Zhang C, Xie Y, Sun X, Liu G, Deng C: Intratunical injection of human urine-derived stem cells derived exosomes prevents fibrosis and improves erectile function in a rat model of Peyronie's disease. Andrologia 2020, 52(11):e13831.

8. Dong X, Zhang T, Liu Q, Zhu J, Zhao J, Li J, Sun B, Ding G, Hu X, Yang $Z$ et al: Beneficial effects of urine-derived stem cells on fibrosis and apoptosis of myocardial, glomerular and bladder cells. Molecular and cellular endocrinology 2016, 427:21-32.

9. Yao Z, Li J, Wang X, Peng S, Ning J, Qian Y, Fan C: MicroRNA-21-3p Engineered Umbilical Cord Stem Cell-Derived Exosomes Inhibit Tendon Adhesion. Journal of inflammation research 2020, 13:303-316.

10. Shao L, Zhang Y, Pan X, Liu B, Liang C, Zhang Y, Wang Y, Yan B, Xie W, Sun $Y$ et al: Knockout of beta-2 microglobulin enhances cardiac repair by modulating exosome imprinting and inhibiting stem cell-induced immune rejection. Cellular and molecular life sciences: CMLS 2020, 77(5):937-952.

11. Zhang DH, Zhang JL, Huang Z, Wu LM, Wang ZM, Li YP, Tian XY, Kong LY, Yao R, Zhang YZ: Deubiquitinase Ubiquitin-Specific Protease 10 Deficiency Regulates Sirt6 signaling and Exacerbates Cardiac Hypertrophy. Journal of the American Heart Association 2020, 
9(22):e017751.

12. Li Y, Liu M, Song X, Zheng X, Yi J, Liu D, Wang S, Chu C, Yang J: Exogenous Hydrogen Sulfide Ameliorates Diabetic Myocardial Fibrosis by Inhibiting Cell Aging Through SIRT6/AMPK Autophagy. Frontiers in pharmacology 2020, 11:1150.

13. Zhang Q, Tu W, Tian K, Han L, Wang Q, Chen P, Zhou X: Sirtuin 6 inhibits myofibroblast differentiation via inactivating transforming growth factor- $\beta 1 /$ Smad 2 and nuclear factor-kB signaling pathways in human fetal lung fibroblasts. Journal of cellular biochemistry 2019, 120(1):93-104.

14. Tian K, Chen P, Liu Z, Si S, Zhang Q, Mou Y, Han L, Wang Q, Zhou X: Sirtuin 6 inhibits epithelial to mesenchymal transition during idiopathic pulmonary fibrosis via inactivating TGF- $\beta$ 1/Smad3 signaling. Oncotarget 2017, 8(37):61011-61024.

15. Zhong X, Huang M, Kim HG, Zhang Y, Chowdhury K, Cai W, Saxena R, Schwabe RF, Liangpunsakul S, Dong XC: SIRT6 Protects Against Liver Fibrosis by Deacetylation and Suppression of SMAD3 in Hepatic Stellate Cells. Cellular and molecular gastroenterology and hepatology 2020, 10(2):341-364.

16. Zhang J, Li Y, Liu Q, Huang Y, Li R, Wu T, Zhang Z, Zhou J, Huang H, Tang $Q$ et al: Sirt6 Alleviated Liver Fibrosis by Deacetylating Conserved Lysine 54 on Smad2 in Hepatic Stellate Cells. Hepatology (Baltimore, Md) 2020.

17. Huang W, Liu H, Zhu S, Woodson M, Liu R, Tilton RG, Miller JD, Zhang W: Sirt6 deficiency results in progression of glomerular injury in the renal. Aging 2017, 9(3):1069-1083.

18. Cai J, Liu Z, Huang X, Shu S, Hu X, Zheng M, Tang C, Liu Y, Chen G, Sun $L$ et al: The deacetylase sirtuin 6 protects against renal fibrosis by epigenetically blocking $\beta$-catenin target gene expression. Renal international 2020, 97(1):106-118.

19. Gu Y-Y, Liu X-S, Huang X-R, Yu X-Q, Lan H-Y: Diverse Role of TGF- $\beta$ in Renal Disease. Front Cell Dev Biol 2020, 8:123.

20. Meng X-M, Nikolic-Paterson DJ, Lan HY: TGF- $\beta$ : the master regulator of fibrosis. Nat Rev Nephrol 2016, 12(6):325-338.

21. Yao $Y$, Chen R, Wang G, Zhang Y, Liu F: Exosomes derived from mesenchymal stem cells reverse EMT via TGF- $\beta 1 /$ Smad pathway and promote repair of damaged endometrium. Stem Cell Res Ther 2019, 10(1):225. 